\title{
Specific Diagnosis of Two Root-Knot Nematodes, Meloidogyne chitwoodi and M. fallax, with Satellite DNA Probes
}

\author{
P. Castagnone-Sereno, F. Leroy, M. Bongiovanni, C. Zijlstra, and P. Abad
}

First, second, third, and fifth authors: INRA, Unité Santé Végétale et Environnement, BP 2078, 06606 Antibes Cedex, France; and fourth author: IPO-DLO, Research Institute for Plant Protection, $6700 \mathrm{GW}$ Wageningen, the Netherlands. Accepted for publication 28 January 1999.

\begin{abstract}
Castagnone-Sereno, P., Leroy, F., Bongiovanni, M., Zijlstra, C., and Abad, P. 1999. Specific diagnosis of two root-knot nematodes, Meloidogyne chitwoodi and M. fallax, with satellite DNA probes. Phytopathology 89: 380-384.

Meloidogyne chitwoodi and M. fallax are serious pests of potato, and both species have been recently designated as quarantine organisms in the European Community and in Canada. The sympatric and less damaging species $M$. hapla is often found associated with both of them under temperate climates. Here, we describe the use of satellite DNA (satDNA)

M. fallax from M. hapla using satDNA monomers as probes. In squashblot experiments, satDNAs allowed discrimination between single individuals of M. chitwoodi or M. fallax from M. hapla, even within root tissues, without the need for DNA purification. The same results were obtained with radioactive or digoxigenin-labeled probes with no loss of sensitivity in detection. M. fallax and M. chitwoodi could not be distinguished. From this study, it is concluded that such cloned satDNA sequences may constitute a powerful tool for the identification and management of Meloidogyne spp. populations in the field and for the implementation of quarantine regulations against these pests.
\end{abstract} sequences previously isolated from these three root-knot nematode species for the development of specific diagnostic procedures. In dot-blot experiments, it was unambiguously possible to separate $M$. chitwoodi and
Additional keywords: species-specific probe, squash-blot procedure, tandem repeats.
The root-knot nematode species Meloidogyne chitwoodi Golden, O'Bannon, Santo, \& Finley and M. fallax Karssen are important pathogens of potato and other crops in the western part of Europe, where a significant increase of the populations has been observed recently (12). M. chitwoodi is also a major pest of potato in the northwestern states of the United States, where M. fallax has not yet been reported (13). These nematodes cause severe galling on the surface and necrotic spotting below the skin of the tubers, which become unmarketable for either fresh consumption or processing. Because of their current emergence as serious pests, $M$. chitwoodi and $M$. fallax have recently been designated as quarantine organisms in the European Community and in Canada (1,14). M. hapla Chitwood, which causes less economic damage on potato than does M. chitwoodi or M. fallax (13), is very often found associated with both species under temperate climates. Because the three species resemble each other morphologically, simple and reliable identification tools for these Meloidogyne spp. are urgently needed for both the identification and management of populations in the field and for the implementation of quarantine regulations by official plant protection services.

Recently, different methodologies based on the polymerase chain reaction (PCR) have been developed to identify these root-knot nematode species. Restriction analysis of PCR-amplified ribosomal DNA (rDNA) sequences containing the internal transcribed spacer (ITS) was successfully used to distinguish between M. hapla and $M$. chitwoodi isolates (21). In the same way, amplification of the intergenic spacer (IGS) region of the genomic rDNA produced polymorphic fragments between $M$. hapla, M. chitwoodi, and $M$. fallax, although the size difference of the amplified bands was very low between $M$. chitwoodi and $M$. fallax (13). Design of specific primers derived from the sequence of the amplified ITS re-

Corresponding author: P. Castagnone-Sereno; E-mail address: pca@ antibes.inra.fr

Publication no. P-1999-0305-01R

(C) 1999 The American Phytopathological Society gion resulted in fragment-size polymorphisms that distinguish the three species, without the need for subsequent restriction analysis (20). The same results were also obtained with primers specific for the IGS region of nematode rDNA (15).

Satellite DNA (satDNA) is present in the genome of almost all eukaryotic organisms and is composed of highly repetitive sequences organized as long arrays of tandemly repeated elements, present from $10^{3}$ to $10^{5}$ copies per haploid genome (2). Recently, satDNA sequences have been characterized from a number of plant-parasitic and entomopathogenic nematodes and have proven to be species specific, thus constituting useful diagnostic tools for the identification of species of agronomic interest (9). Therefore, an alternative strategy for specific identification that does not involve PCR amplification of nematode DNA could be considered in the case of $M$. chitwoodi and M. fallax, based on such sequences. A StyI satDNA family was isolated from M. hapla (16), and its use as a probe demonstrated that it does not hybridize with the genomic DNA from $M$. chitwoodi isolates (17). Very recently, we cloned and characterized two $A l u$ I satDNA families from the genome of $M$. chitwoodi and $M$. fallax, respectively $(4,5)$. The objectives of the study reported here were to evaluate the species specificity of both sequences, compared with $M$. hapla, and then to design a diagnostic procedure that could be easily manipulated on a routine basis.

\section{MATERIALS AND METHODS}

Nematode isolates. Root-knot nematode isolates originating from Europe, the United States, and Canada (Table 1) were maintained on tomatoes (Lycopersicon esculentum L. cv. Moneymaker) grown in a sand-peat mixture in $1,000-\mathrm{cm}^{3}$ clay pots at $20^{\circ} \mathrm{C}$ in a greenhouse. They were specifically identified morphologically, according to their isoesterase electrophoretic pattern, or both (7).

DNA isolation. Eggs were extracted from Meloidogyne spp.-infested roots, collected (10), and placed on a 10- $\mu$ m-pore sieve at $20^{\circ} \mathrm{C}$ for 3 weeks to allow the eggs to hatch. Second-stage juveniles were collected every week and concentrated by centrifugation 
at $2,000 \times g$ for 2 min in a $30 \%$ sucrose solution, washed in distilled water, and pelleted in a microcentrifuge. The pellet was transferred to a mortar, frozen by liquid nitrogen, and ground. From the resulting fine powder, total DNA was extracted as described by Curran et al. (6), resuspended in Tris-EDTA (TE) buffer (0.01 M Tris, $\mathrm{pH}$ 8.0, and $0.001 \mathrm{M}$ EDTA), and stored at $-80^{\circ} \mathrm{C}$ until use.

DNA probes. Three different DNA probes were used in the experiments: pMcCo14 (GenBank accession number U96668), pMfFd1.9 (EMBL accession number AJ223457), and pMhM6 (GenBank accession number L07109). They consisted of cloned monomers of satDNAs previously identified in $M$. chitwoodi, $M$. fallax, and $M$. hapla, respectively $(4,5,16)$.

TABLE 1. Meloidogyne spp. and isolates used in this study

\begin{tabular}{|c|c|c|c|}
\hline Isolates & Origin $^{\mathrm{a}}$ & Source $^{b}$ & Last host \\
\hline \multicolumn{4}{|l|}{ M. chitwoodi } \\
\hline $\mathrm{Ca}$ & The Netherlands (SE) & PD & Maize \\
\hline $\mathrm{Ci}$ & The Netherlands (SE) & PD & Tomato \\
\hline $\mathrm{Cj}$ & The Netherlands (SE) & PD & Unknown \\
\hline $\mathrm{Cl}$ & The Netherlands (NE) & PD & Wheat \\
\hline $\mathrm{Cp}$ & The Netherlands (SE) & PAV & Black salsify \\
\hline $\mathrm{Cu}$ & The Netherlands (SE) & PD & Potato \\
\hline $\mathrm{Cx}$ & The Netherlands (S) & PD & Potato \\
\hline $\mathrm{Cy}$ & The Netherlands (SE) & PD & Potato \\
\hline $\mathrm{Cz}$ & The Netherlands (SE) & PD & Potato \\
\hline Caj & The Netherlands (SE) & PD & Potato \\
\hline $\mathrm{Caq}$ & The Netherlands & PD & Potato \\
\hline Cav & Washington & WSU & Potato \\
\hline Cba (race 1) & Oregon & WSU & Potato \\
\hline Cbd (race 2) & Washington & WSU & Potato \\
\hline Cbh (race 3) & Tulelake, California & WSU & Potato \\
\hline Cbj & The Netherlands (SE) & BLGG & Unknown \\
\hline Cbk & The Netherlands (SE) & BLGG & Unknown \\
\hline Cbn & The Netherlands (SE) & BLGG & Unknown \\
\hline Cbo & The Netherlands (SE) & BLGG & Unknown \\
\hline Cbs & The Netherlands (NE) & PAV & Unknown \\
\hline $\mathrm{Cbu}$ & The Netherlands (NE) & PAV & Unknown \\
\hline $\mathrm{Cbv}$ & The Netherlands (NE) & PAV & Unknown \\
\hline $\mathrm{Cbz}$ & The Netherlands (NE) & PAV & Unknown \\
\hline $\mathrm{Ccc}$ & The Netherlands (SW) & $\mathrm{PD}$ & Onion \\
\hline \multicolumn{4}{|l|}{ M. fallax } \\
\hline $\mathrm{Fa}$ & The Netherlands (SE) & PAV & Primrose \\
\hline $\mathrm{Fb}$ & The Netherlands (SE) & PD & Beet \\
\hline $\mathrm{Fc}$ & The Netherlands (SE) & PD & Potato \\
\hline $\mathrm{Fd}$ & The Netherlands (SE) & PD & Potato \\
\hline $\mathrm{Fe}$ & The Netherlands (SW) & BLGG & Unknown \\
\hline Ff & Belgium & ARC & Unknown \\
\hline $\mathrm{Fg}$ & The Netherlands(S) & CPRO & Unknown \\
\hline \multicolumn{4}{|l|}{ M. hapla } \\
\hline $\mathrm{Ha}$ & The Netherlands (W) & PD & Peony \\
\hline $\mathrm{Hc}$ & The Netherlands (S) & PD & Aconitum sp. \\
\hline $\mathrm{He}$ & The Netherlands (NE) & PAV & Potato \\
\hline Hp & The Netherlands & HLB & Potato \\
\hline $\mathrm{Hau}$ & Utah & WSU & Potato \\
\hline AN2 & La Môle, France & INRA & Grape \\
\hline AN3 & England & INRA & Unknown \\
\hline AN4 & Frontignan, France & INRA & Unknown \\
\hline $\mathrm{Hbg}$ & Wyoming & UW & Unknown \\
\hline $\mathrm{Hbj}$ & Canada & UW & Unknown \\
\hline $\mathrm{Hbq}$ & Hungary & PD & Rose \\
\hline Hbr & The Netherlands (NW) & PD & Veronica sp. \\
\hline Hbs & The Netherlands (SE) & PD & Fennel \\
\hline $\mathrm{Hbv}$ & The Netherlands & BLGG & Rose \\
\hline $\mathrm{Hcb}$ & The Netherlands & CPRO & Hemp \\
\hline
\end{tabular}

a Parts of the Netherlands: $\mathrm{SE}=$ southeastern, $\mathrm{S}=$ southern, $\mathrm{NE}=$ northeastern, $\mathrm{SW}=$ southwestern, and $\mathrm{NW}=$ northwestern.

${ }^{\mathrm{b}} \mathrm{PD}=$ Plant Protection Service, Wageningen, the Netherlands; PAV $=$ Research Station for Arable Farming and Field Production of Vegetables, Lelystad, the Netherlands; WSU = Washington State University, Prosser; BLGG = Laboratory for Soil and Crop Testing, Oosterbeek, the Netherlands; ARC = Agricultural Research Centre, Merelbeke, Belgium; $\mathrm{CPRO}=$ Center for Plant Breeding and Reproduction Research, Wageningen, the Netherlands; HLB = Hillbrands Laboratorium, Assen, the Netherlands; INRA = Institut National de la Recherche Agronomique, Antibes, France; and UW = University of Wisconsin, Madison.
Radioactive labeling and hybridization conditions. DNA probes were radioactively labeled according to the random oligonucleotide priming method (8) with alpha- ${ }^{32} \mathrm{P}$ dCTP. Hybridizations were conducted at high stringency $(6 \times \mathrm{SSC}[1 \times \mathrm{SSC}$ is $0.15 \mathrm{M}$ $\mathrm{NaCl}$ plus $0.015 \mathrm{M}$ sodium citrate], $5 \times$ Denhardt's, and $0.5 \%$ sodium dodecyl sulfate [SDS] at $65^{\circ} \mathrm{C}$ ). After hybridization, filters were washed at $65^{\circ} \mathrm{C}$ in $2 \times \mathrm{SSC}$ and $0.1 \%$ SDS, and then in $1 \times \mathrm{SSC}$ and $0.1 \%$ SDS. After posthybridization washes, filters were exposed to X-ray film with an intensifying screen at $-80^{\circ} \mathrm{C}$.

Digoxigenin (DIG) labeling, hybridization, and detection conditions. PCR was used to produce probes directly labeled with DIG using the PCR DIG Labeling Mix (Boehringer GmbH, Mannheim, Germany) that contains a 19:1 ratio of dTTP:DIG-dUTP, according to the manufacturer's instructions. The primers used are show in Table 2, and the thermal profile was as follows: 3 min at $94^{\circ} \mathrm{C}$ and then 35 cycles of denaturation at $94^{\circ} \mathrm{C}$ for $1 \mathrm{~min}$, annealing at $52^{\circ} \mathrm{C}$ for $1 \mathrm{~min}$, and extension at $72^{\circ} \mathrm{C}$ for $1 \mathrm{~min}$. A 10 -min incubation period at $72^{\circ} \mathrm{C}$ followed the last cycle to complete any partially synthesized strand.

Membranes were prehybridized for $1 \mathrm{~h}$ at $65^{\circ} \mathrm{C}$ in hybridization buffer $(2.5 \times$ SSC, $0.01 \%$ SDS, $0.1 \% N$-lauroylsarcosine, and $1 \%$ blocking reagent) and then incubated overnight at $65^{\circ} \mathrm{C}$ in the same buffer in which the appropriate probe had been added. After hybridization, membranes were successively washed at $65^{\circ} \mathrm{C}$ in $2 \times \mathrm{SSC}$ and $0.1 \%$ SDS (30 min), in $0.2 \times$ SSC and $0.1 \% \operatorname{SDS}(30 \mathrm{~min})$, and finally in washing buffer $(0.1 \mathrm{M}$ maleic acid, $0.15 \mathrm{M} \mathrm{NaCl}$, and $0.3 \%$ [wt/vol] Tween 20, pH 7.5). Membranes were then incubated for $1 \mathrm{~h}$ in blocking buffer (10\% blocking reagent [wt/vol] in $0.1 \mathrm{M}$ maleic acid and $0.15 \mathrm{M} \mathrm{NaCl}, \mathrm{pH} 7.5)$. For detection, the anti-DIG-alkaline phosphatase (AP) conjugate (Fab fragments from an anti-DIG antibody from sheep, conjugated with AP) was diluted $1: 40,000$ in blocking buffer, and the membranes were incubated in this solution for $30 \mathrm{~min}$. For the detection procedure, membranes were rinsed in washing buffer (15 min, twice) and incubated for $5 \mathrm{~min}$ in detection buffer (0.1 M Tris- $\mathrm{HCl}$ and $0.1 \mathrm{M} \mathrm{NaCl}, \mathrm{pH} 9.5)$. The CDP-star solution (Boehringer $\mathrm{GmbH}$ ), an ultra-sensitive chlorosubstituted 1,2-dioxetane chemiluminescent substrate for AP, was diluted 1:200 in $2 \mathrm{ml}$ of detection buffer, and the membranes were incubated in this solution for $5 \mathrm{~min}$ at room temperature. Membranes were then drained on paper and exposed to X-ray film with intensifying screen at room temperature.

Dot-blot procedure. Dot blots of genomic DNA were prepared by first diluting the DNA to the required concentration $(10 \mathrm{ng} / \mu \mathrm{l})$, denaturing it with $2 \mathrm{M} \mathrm{NaOH}$, and finally neutralizing it with $4 \mathrm{M}$ ammonium acetate. The samples (25 ng of DNA) were then dotted by vacuum onto nitrocellulose membranes in a MiniFold I blotting apparatus (Schleicher \& Schuell, Dassel, Germany). Each dot-blot experiment was repeated at least twice.

Squashed nematode experiment. The experiment was conducted on isolates AN2, Cy, and Fa for M. hapla, M. chitwoodi, and $M$. fallax, respectively. For each isolate, one female, five females, one egg mass, and one gall were hand-picked and placed on a nylon membrane. The nematodes were then ruptured by gentle pressure with a yellow, flat-tipped micropipette tip. Squashed materials were lysed by layering, successively, the filter on Whatman 3MM paper (Whatman International Ltd., Maidstone, England) soaked with $10 \%$ SDS ( $2 \mathrm{~min}$ ), $0.5 \mathrm{M} \mathrm{NaOH}$ and $2.5 \mathrm{M} \mathrm{NaCl}$ (twice, $5 \mathrm{~min}$ each), and $3 \mathrm{M}$ sodium acetate, $\mathrm{pH} 5$ (three times, $2 \mathrm{~min}$ each). The filter was dried at room temperature $(30 \mathrm{~min})$ and then

TABLE 2. Primers used in this study

\begin{tabular}{lll}
\hline Species & Code & \multicolumn{1}{c}{ Sequence (5' to 3') } \\
\hline M. chitwoodi & McS1 & CTTGTTCGATTCACCTCTTCATCCTC \\
& McS2 & CTATGAAATTCAAAAATC \\
M. fallax & MfX1 & AGAATGAAGATTTTTGAAG \\
& MfX2 & AGCTTCATTATATTCAGAAT \\
M. hapla & MhS1 & GAGTTCCTTGTAAAGCAACTTCT \\
& MhS2 & GGACTTTTTAGGACACAGC \\
\hline
\end{tabular}


baked at $80^{\circ} \mathrm{C}$ for $1 \mathrm{~h}$. Prehybridization and hybridization with the DIG-labeled probes were carried out as described above. The whole procedure was carried out three times to verify reproducibility.

\section{RESULTS}

Use of pMcCo and pMfFd repetitive sequences as diagnostic probes. The structural characteristics of the AluI repetitive sequences $\mathrm{pMcCo}$ and $\mathrm{pMfFd}$, isolated from the genome of $M$. chitwoodi and $M$. fallax, respectively, have been described previously $(4,5)$. To test their distribution, the genomic DNA of $22 M$. chitwoodi isolates, $7 \mathrm{M}$. fallax isolates, and $13 \mathrm{M}$. hapla isolates was dot-blotted on a nylon membrane and hybridized first with a radioactively labeled monomer from the pMcCo satDNA. Hybridization occurred with all the $M$. chitwoodi and M. fallax populations and never with any of the M. hapla populations (Fig. 1A).
After dehybridization, the same membrane was hybridized again using a monomer of the pMfFd satellite family cloned in M. fallax, and the results were absolutely similar to those obtained with the probe isolated from $M$. chitwoodi (Fig. 1B). These results demonstrated that both satDNAs are able to separate M. chitwoodi and M. fallax from the sympatric species M. hapla.

DIG labeling of the probes. It is clear that radioactive compounds are not suitable for a routine diagnostic protocol. Therefore, we initiated experiments on a subset of nematode populations in order to test the "cold" labeling of the probes with DIG. In addition to the two previous satDNAs, pMhM, a StyI satDNA cloned in $M$. hapla, was used because its distribution was restricted to this root-knot nematode species only $(3,16,17)$. The three probes were PCR-labeled with DIG and used to successively hybridize a dot blot containing genomic DNAs from populations of $M$. hapla, M. chitwoodi, and M. fallax. Use of pMcCo and pMfFd gave the
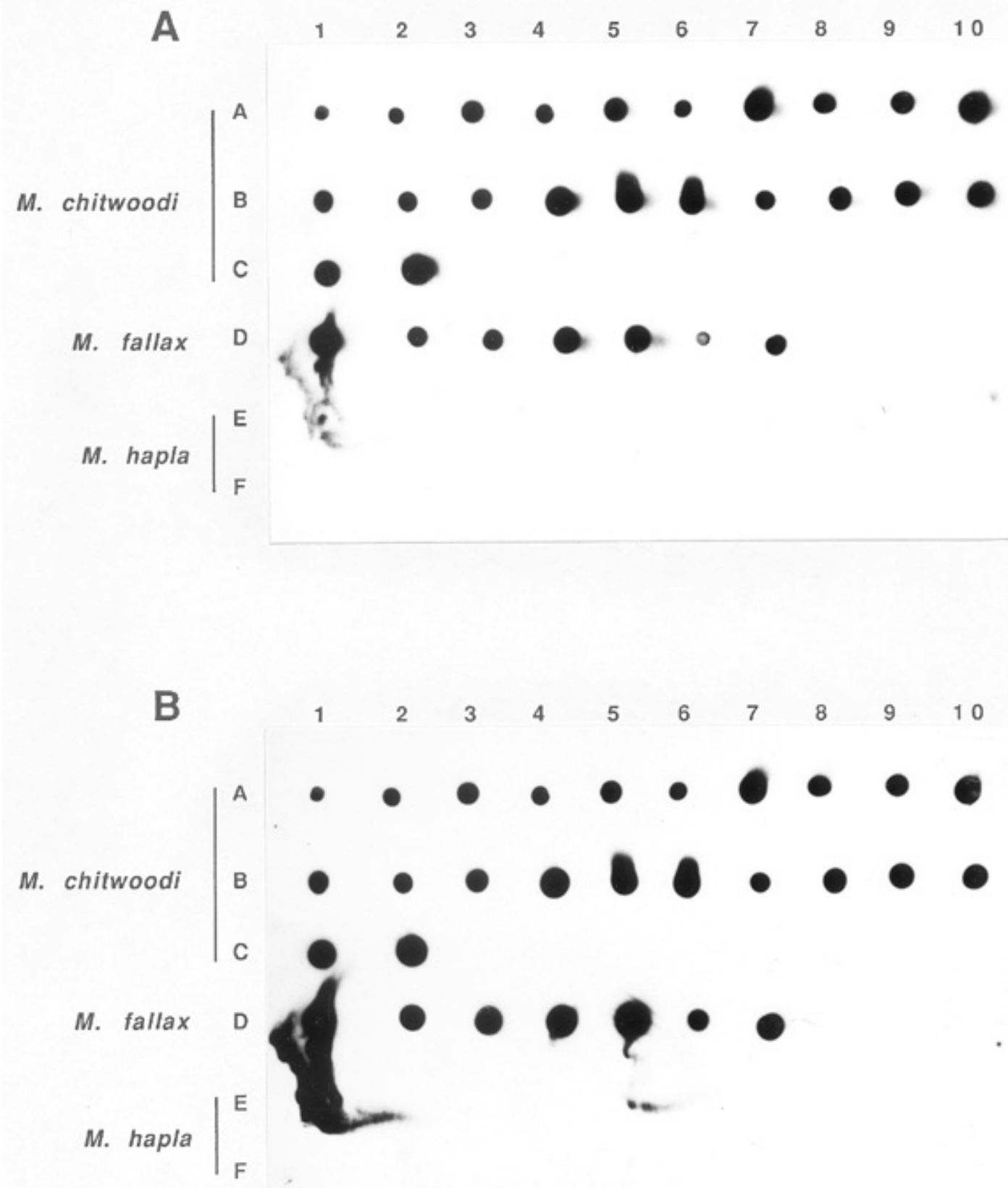

Fig. 1. Dot-blot experiments. A dot-blot of Meloidogyne chitwoodi, M. fallax, and M. hapla populations was successfully hybridized with an alpha- ${ }^{32} \mathrm{P}-\mathrm{labeled}$ satellite DNA probe from $\mathbf{A}, M$. chitwoodi and $\mathbf{B}, M$. fallax. Population codes are $M$. chitwoodi isolates $\mathrm{Ca}=\mathrm{A} 1, \mathrm{Ci}=\mathrm{A} 2, \mathrm{Cj}=\mathrm{A} 3, \mathrm{Cl}=\mathrm{A} 4, \mathrm{Cu}=\mathrm{A} 5, \mathrm{Cx}=\mathrm{A} 6$, $\mathrm{Cy}=\mathrm{A} 7, \mathrm{Cz}=\mathrm{A} 8, \mathrm{Caj}=\mathrm{A} 9, \mathrm{Caq}=\mathrm{A} 10, \mathrm{Cba}($ race 1) $=\mathrm{B} 1, \mathrm{Cbd}($ race 2) $=\mathrm{B} 2, \mathrm{Cbh}($ race 3$)=\mathrm{B} 3, \mathrm{Cbj}=\mathrm{B} 4, \mathrm{Cbk}=\mathrm{B} 5, \mathrm{Cbn}=\mathrm{B} 6, \mathrm{Cbo}=\mathrm{B} 7, \mathrm{Cbs}=\mathrm{B} 8, \mathrm{Cbu}=$ $\mathrm{B} 9, \mathrm{Cbv}=\mathrm{B} 10, \mathrm{Cbz}=\mathrm{C} 1$, and $\mathrm{Ccc}=\mathrm{C} 2 ;$. fallax isolates $\mathrm{Fa}=\mathrm{D} 1, \mathrm{Fb}=\mathrm{D} 2, \mathrm{Fc}=\mathrm{D} 3, \mathrm{Fd}=\mathrm{D} 4, \mathrm{Fe}=\mathrm{D} 5, \mathrm{Ff}=\mathrm{D} 6$, and $\mathrm{Fg}=\mathrm{D} 7$; and $M$. hapla isolates $\mathrm{Ha}=$ $\mathrm{E} 1, \mathrm{Hc}=\mathrm{F} 2, \mathrm{He}=\mathrm{F} 1, \mathrm{Hp}=\mathrm{E} 3, \mathrm{AN} 2=\mathrm{E} 10, \mathrm{AN} 3=\mathrm{E} 9, \mathrm{Hbj}=\mathrm{E} 8, \mathrm{Hbq}=\mathrm{E} 7, \mathrm{Hbr}=\mathrm{E} 6, \mathrm{Hbs}=\mathrm{E} 5, \mathrm{Hbv}=\mathrm{E} 4$, and $\mathrm{Hcb}=\mathrm{E} 2$. 
same hybridization patterns as previously, thus clearly separating $M$. chitwoodi and M. fallax on the one hand, and M. hapla on the other hand (Fig. 2A and B). Complementarily, pMhM hybridized specifically with genomic DNAs from the M. hapla populations

\section{$\begin{array}{llllll}\text { A } & 1 & 2 & 3 & 4 & 5\end{array}$ \\ M. chitwoodi \\ A

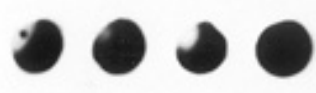 \\ M. fallax \\ B

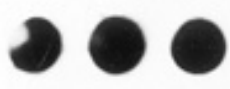

M. hapla

C

M. chitwoodi

A

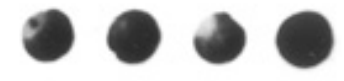

M. fallax

B

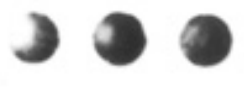

M. hapla

C

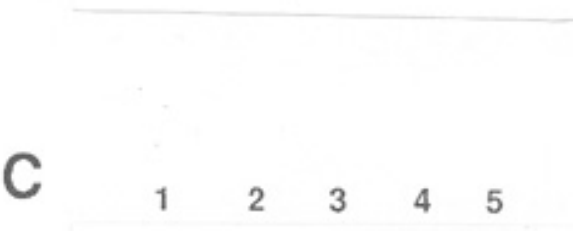

M. chitwoodi

A

M. fallax
M. hapla

C

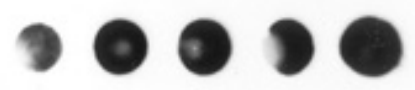

Fig. 2. Dot-blot experiments. A dot-blot of Meloidogyne chitwoodi, M. fallax, and $M$. hapla populations was successfully hybridized with a digoxigenin-labeled satellite DNA probe from A, M. chitwoodi; B, M. fallax; and C, $M$. hapla. Population codes are $M$. chitwoodi isolates $\mathrm{Cp}=\mathrm{B} 3, \mathrm{Cav}=\mathrm{B} 1$, and $\mathrm{Cbd}($ race 2) $=\mathrm{B} 2 ; M$. fallax isolates $\mathrm{Fa}=\mathrm{A} 1, \mathrm{Fc}=\mathrm{A} 2, \mathrm{Fd}=\mathrm{A} 3$, and $\mathrm{Fe}=$ $\mathrm{A} 4$; and $M$. hapla isolates $\mathrm{Hau}=\mathrm{C} 1, \mathrm{AN} 2=\mathrm{C} 3, \mathrm{AN} 3=\mathrm{C} 4, \mathrm{AN} 4=\mathrm{C} 5$, and $\mathrm{Hbg}=\mathrm{C} 2$. only (Fig. 2C). Due to the repetitive nature of satDNA, no loss of sensitivity in the detection was observed with the cold probes compared with the radioactive ones. Moreover, the exposure time needed to obtain a readable hybridization signal were considerably reduced with the DIG-labeled probes (from $30 \mathrm{~min}$ to $1 \mathrm{~h}$ ) compared with the radioactively labeled ones (12 to $24 \mathrm{~h}$ ).

Detection of single individuals in squashed nematode experiments. The restricted distribution of the three satDNA families, and also their high level of reiteration in the genome, allowed us to test them as hybridization probes in squashed nematode experiments. The theoretical advantage of such a procedure is that it should work directly on the biological material, without the need for any time-consuming DNA purification step. Once more, probes were PCR-labeled with DIG. Either one single female, one egg mass, or one female in galled root tissues could unambiguously be identified with the corresponding satDNA probe after a 1-h ex-

A

$\begin{array}{cccc}\text { galled } & 1 \text { egg } & 5 & 1 \\ \text { root } & \text { mass } & \text { females } & \text { female }\end{array}$

M. chitwoodi

M. fallax
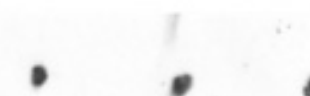

M. hapla

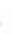

B

M. chitwoodi

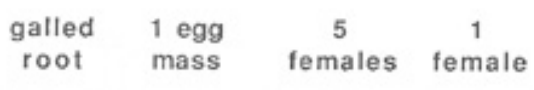

M. fallax

M. hapla
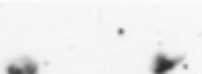

C

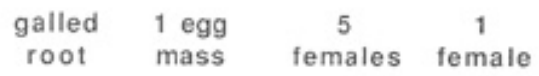

M. chitwoodi

M. fallax

M. hapla

Fig. 3. Squashed nematode experiments. Squashed Meloidogyne chitwoodi, M. fallax, and M. hapla materials were successfully hybridized with a digoxigenin-labeled satellite DNA probe from A, M. chitwoodi; B, M. fallax; and C, M. hapla. 
posure of the membrane, with the same specificity as displayed in both previous dot-blot experiments (Fig. 3). As expected, hybridization of individuals from other root-knot nematode species and of healthy root material gave no signal (data not shown).

\section{DISCUSSION}

Repetitive sequences known as satDNA have been characterized in a number of nematodes of agronomic interest, and because of their species specificity and reiteration in the genome, they have provided very powerful tools to discriminate between closely related species for which specific markers are needed (9). Here, we present evidence that such sequences may be of interest for the development of a routine diagnostic procedure for the identification of the quarantine root-knot nematodes $M$. chitwoodi and $M$. fallax versus the sympatric species $M$. hapla. Using previously cloned satDNAs from these three species $(4,5,16)$ as probes in squash-blot experiments, it was possible to unambiguously discriminate between single individuals of $M$. chitwoodi and M. fallax on one side and of $M$. hapla on the other, even within root tissues, without the need for either DNA purification or radioactive compounds for the labeling of the probes.

The monomeric unit of the satDNA from $M$. hapla hybridized only with genomic DNA from populations belonging to this species, without any cross-hybridization observed with populations from either $M$. chitwoodi or $M$. fallax. This specific distribution, inferred from the analysis of a large collection of populations, thus confirmed previous results obtained on a more restricted number of populations (17).

Cross-hybridizations were observed between the satDNA sequences from either $M$. chitwoodi or M. fallax and the genomic DNAs of these two species. Because of the usual species restricted distribution of satDNA (9), this result could be considered as unexpected. However, it is easily related to some recent taxonomical, biological, and molecular data. Due to morphological resemblance, $M$. fallax had originally been misidentified as a new race of $M$. chitwoodi (19) and was recognized as a new root-knot nematode species only recently (11). Moreover, successful interspecific hybridization was obtained between the two species, which provided strong evidence supporting their close phylogenetic relationships (18). From a molecular point of view, pairwise sequence comparison of consensus sequences revealed a high sequence similarity $(68.39 \%)$ between the satDNA from $M$. fallax and satDNA subfamily $2 \mathrm{a}$ of $M$. chitwoodi (5). From these data, it was hypothesized that $M$. fallax and $M$. chitwoodi may share a common ancestor and that $M$. fallax could be the result of a recent speciation event. Therefore, the cross-hybridization observed in the current experiment could reflect some ancestral subsequence common to both $M$. fallax and M. chitwoodi.

From a practical point of view, the squashed nematode procedure described here presents technical features that should be of interest for the development of a routine diagnostic test for the identification of $M$. chitwoodi and $M$. fallax versus the sympatric species M. hapla. First, the use of a nonradioactive labeling procedure is safe compared with the radioactive compounds generally used in hybridization experiments. Second, after hybridization against the blotted target, the hybridization solution still contains large amounts of unannealed DIG-labeled probe, which is stable for at least 1 year when stored at $-20^{\circ} \mathrm{C}$ and can be reused several times. Third, because of the repetitive nature of satDNA, the probe can detect one single nematode, whatever its developmental stage (e.g., eggs and juveniles in egg masses; and females, even in root tissues) and without the need for any time-consuming DNA purification step. Therefore, it appears from this study that cloned satDNAs may constitute a powerful tool for the identification and management of Meloidogyne spp. populations in the field and for the implementation of quarantine regulations against these pests.

\section{ACKNOWLEDGMENTS}

This research was financially supported by the Commission of the European Community (contract FAIR1-CT95-0896). We thank G. J. W. Janssen, A. MacGuidwin, M. Moens, H. Mojtahedi, L. Molendijk, G. Santo, G. Veninga, and the organizations ARC, Merelbeke, Belgium; BLGG, Oosterbeek, the Netherlands; CPRO-DLO, Wageningen, the Netherlands; HLB, Assen, the Netherlands; PAV, Lelystad, the Netherlands; PD, Wageningen, the Netherlands; University of Wisconsin, Madison; and Washington State University, Prosser, for providing nematode isolates; and C. Slagmulder for the photographic artwork.

\section{LITERATURE CITED}

1. Anonymous. 1998. Directive $98 / 1 / \mathrm{CE}$ de la Commission du 8 Janvier 1998. J. Off. Commun. Eur. L15:26-33.

2. Beridze, T. G. 1986. Satellite DNAs. Springer-Verlag, Berlin.

3. Castagnone-Sereno, P., Esparrago, G., Abad, P., Leroy, F., and Bongiovanni, M. 1995. Satellite DNA as a target for PCR-specific detection of the plantparasitic nematode Meloidogyne hapla. Curr. Genet. 28:566-570.

4. Castagnone-Sereno, P., Leroy, H., Semblat, J. P., Leroy, F., Abad, P., and Zijlstra, C. 1998. Unusual and strongly structured sequence variation in a complex satellite DNA family from the nematode Meloidogyne chitwoodi. J. Mol. Evol. 46:225-233.

5. Castagnone-Sereno, P., Semblat, J. P., Leroy, F., and Abad, P. 1998. A new AluI satellite DNA in the root-knot nematode Meloidogyne fallax: Relationships with satellites from the sympatric species $M$. hapla and $M$. chitwoodi. Mol. Biol. Evol. 15:1115-1122.

6. Curran, J., McClure, M. A., and Webster, J. M. 1986. Genotypic differentiation of Meloidogyne populations by detection of restriction fragment length difference in total DNA. J. Nematol. 18:83-86.

7. Dalmasso, A., and Bergé, J. B. 1978. Molecular polymorphism and phylogenetic relationship in some Meloidogyne spp.: Application to the taxonomy of Meloidogyne. J. Nematol. 10:323-332.

8. Feinberg, A. P., and Vogelstein, B. 1983. A technique for radiolabeling DNA restriction endonuclease fragments to high specific activity. Anal. Biochem. 112:295-298.

9. Grenier, E., Castagnone-Sereno, P., and Abad, P. 1997. Satellite DNA sequences as taxonomic markers in nematodes of agronomic interest. Parasitol. Today 13:398-401.

10. Hussey, R. S., and Barker, K. R. 1973. A comparison of methods of collecting inocula for Meloidogyne spp., including a new technique. Plant Dis. Rep. 57:1025-1028.

11. Karssen, G. 1996. Description of Meloidogyne fallax n. sp. (Nematoda: Heteroderidae), a root-knot nematode from the Netherlands. Fundam. Appl. Nematol. 19:593-599.

12. Molendijk, L. P. G., and Mulder, A. 1996. The Netherlands, nematodes and potatoes; old problems are here again. Potato Res. 39:471-477.

13. Nyczepir, A. P., Inserra, R. N., O'Bannon, J. H., and Santo, G. S. 1982. Incidence and distinguishing characteristics of Meloidogyne chitwoodi and M. hapla in potato from the northwestern United States. J. Nematol. 14:347-353.

14. Petersen, D. J., and Vrain, T. C. 1996. Rapid identification of Meloidogyne chitwoodi, M. hapla, and M. fallax using PCR primers to amplify their ribosomal intergenic spacer. Fundam. Appl. Nematol. 19:601-605.

15. Petersen, D. J., Zijlstra, C., Wishart, J., Blok, V., and Vrain, T. C. 1997. Specific probes efficiently distinguish root-knot nematode species using signature sequences in the ribosomal intergenic spacer. Fundam. Appl. Nematol. 20:619-626.

16. Piotte, C., Castagnone-Sereno, P., Bongiovanni, M., Dalmasso, A., and Abad, P. 1994. Cloning and characterization of two satellite DNAs in the low-C-value genome of the nematode Meloidogyne spp. Gene 138:175-180.

17. Piotte, C., Castagnone-Sereno, P., Bongiovanni, M., Dalmasso, A., and Abad, P. 1995. Analysis of a satellite DNA from Meloidogyne hapla and its use as a diagnostic probe. Phytopathology 85:458-462.

18. Van der Beek, J. G., and Karssen, G. 1997. Interspecific hybridization of meiotic parthenogenetic Meloidogyne chitwoodi and M. fallax. Phytopathology 87:1061-1066.

19. Van Meggelen, J. C., Karssen, G., Janssen, J. G. W., Verberk-Bakker, B., and Janssen, R. 1994. A new race of Meloidogyne chitwoodi Golden, O’Bannon, Santo \& Finley? Fundam. Appl. Nematol. 17:93-96.

20. Zijlstra, C. 1997. A fast PCR assay to identify Meloidogyne hapla, M. chitwoodi, and M. fallax, and to sensitively differentiate them from each other and from M. incognita in mixtures. Fundam. Appl. Nematol. 20:505-511.

21. Zijlstra, C., Lever, A. E. M., Uenk, B. J., and Van Silfhout, C. H. 1995. Differences between ITS regions of isolates of root-knot nematodes Meloidogyne hapla and M. chitwoodi. Phytopathology 85:1231-1237. 